\title{
Researchers see a need for speed in EU trial approvals
}

In Europe, legislation designed to help harmonize research rules across the continent has failed to improve the time it takes for clinical trials to receive approval, a new analysis suggests. Some experts argue that the measure, known as the European Clinical Trials Directive, has left the continent lagging far behind the United States in this regard and jeopardizes its attractiveness for future research investment.

The new study compared the approval time for clinical trial applications in various nations as part of a single worldwide drug trial. It found the average wait in those EU countries that have implemented the directive was longer than in EU countries still operating under local rules (Br. J. Clin. Pharmacol., doi:10.1111/j.13652125.2008.03246.x; 2008).

Of the fourteen European countries analyzed in the new study, ten had implemented the European Clinical Trials Directive. On average, the approval process took 75 days in the countries under the directive compared with 59 days in the European countries that had still not implemented the directive. There is a theoretical 60-day maximum for authorities to give an opinion under the directive.

Overall, the approvals in Europe took 67 days on average, far longer than the 15 days found in the United States for the same worldwide drug trial.

"The results are surprising," says Dick de Zeeuw, one of the authors of the new study and head of the department of clinical pharmacology at the University Medical Center Groningen in the Netherlands. "I would have expected that in a country where they were applying the directive, it would have been faster."

Concerns over the clinical trials directive, a measure initially adopted by the EU in 2001 to bring research regulations into line and help protect trial subjects, are long standing. For example, a previous analysis in the UK found $62 \%$ of those responding to a government consultation on the legislation worried it could slow or prevent clinical trials (Lancet 362, 1415; 2003).

A key problem is that approval in directive countries runs through local and central ethics committees sequentially rather than simultaneously, say the authors, who urge the EU to provide more guidance on the role of committees and to enforce the 60-day rule.

De Zeeuw cautions that the study only looked at approval of one trial, a 2005 study on a compound called glycosaminoglycan sulodexide for individuals with diabetic kidney disease. But he adds that pharma companies base some of their funding decisions on data such as these, and if the problem of delayed approvals isn't addressed, "it would have a big impact, economically and scientifically".

David Flavell, one of the authors of the earlier UK analysis, told Nature Medicine, "The whole purpose of the [directive] was to make Europe an attractive place for pharma to do clinical trials. Clearly it has not. There's a very real danger there will be a gravitation of clinical trial work away from Europe."

Flavell, a doctor and researcher at the
University of Southampton and scientific director of the charity Leukaemia Busters, said many nonprofits were struggling with the requirements of the directive. Longer approval processes for drugs also present another disincentive to pharma investment in Europe.

"It's very, very difficult to perform multinational, multicenter studies," says a spokeswoman for the European Federation of Pharmaceutical Industries and Associations. "When sponsors need to perform trials quickly, maybe Europe is not the best place to start."

Daniel Cressey, London

\section{Synonym swapping to make vaccines}

Languages abound with synonyms, and people often show preferences for one word over another. Some say 'smart' and others 'sharp' or 'intelligent', but they all mean the same thing. Viruses also have a penchant for certain synonyms, and exploiting these preferences could potentially lead to a new generation of vaccines.

The genetic code consists of threeletter words called codons that specify the amino acids used to build proteins. Typically, more than one codon encodes the same amino acid; for example, GCU, GCC, GCA and GCG are all 'synonymous' codons that represent the amino acid alanine.

Researchers have previously engineered weaker versions of the poliovirus by replacing the virus's favorite codons with its least favorite synonyms (J. Virol. $\mathbf{8 0}$, 3259-3272; 2006). The disliked codons

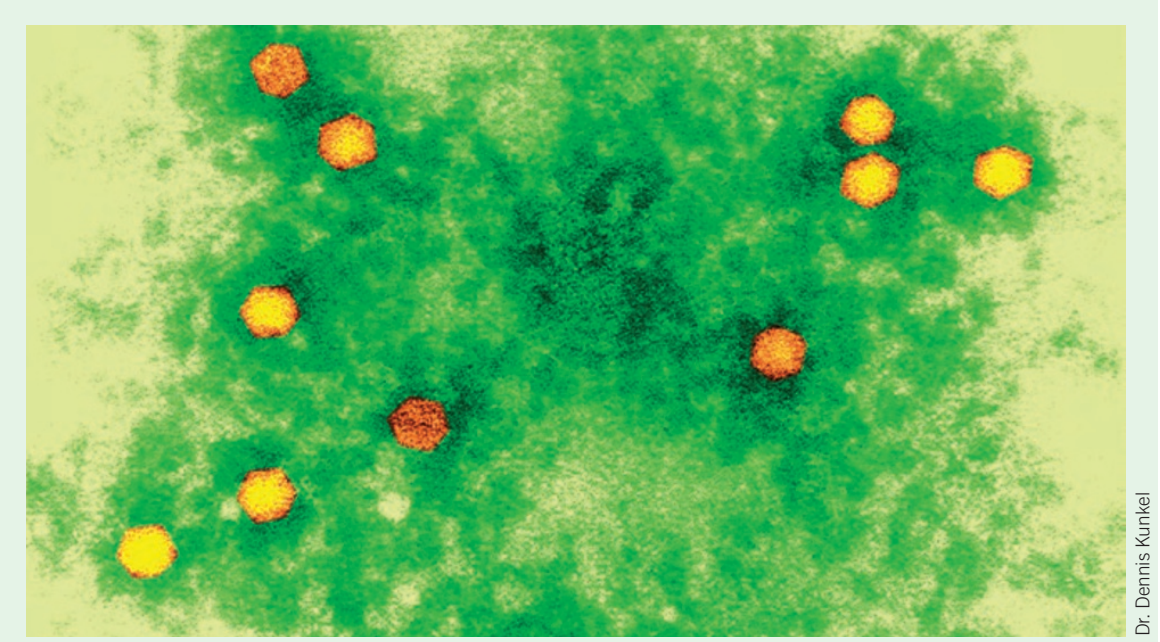

A viral twist: Replacing certain genetic fragments in poliovirus might hold promise seem to act as speed bumps, slowing the rate at which the virus translates its genetic

A team of computer scientists and molecular biologists from Stony Brook University in New York has now used a similar approach to design weakened versions of poliovirus that seem to act as vaccine (Science 320, 1784-1787; 2008). When injected into mice, the engineered viruses helped the animals develop immunity against a normally lethal dose of regular poliovirus.

"We're very interested in trying this in other viruses," says study co-author Steffen Mueller, noting that the approach could potentially be safer and faster than that used for current live vaccine production, which typically requires hazardous starting material (the pathogenic virus).

\section{Coco Ballantyne, New York} code into protein and makes copies of itself. 